\title{
Editorial
}

\section{La Revista de Medicina y Seguridad del Trabajo, su hoy desde el ayer al mañana}

\begin{abstract}
Al emprender un proyecto nos enfrentamos a un reto en el conviven sensaciones de expectativa y de incertidumbre. Al final, sólo el paso del tiempo revela el alcance de sus resultados, pero con total independencia de estos factores, de lo que podemos estar seguros desde el principio hasta el fin, es que sea cual sea el resultado, el esfuerzo siempre ha merecido la pena...
\end{abstract}

...En el verano de 1944, se crea el Instituto Nacional de Medicina y Seguridad del Trabajo, como "un centro técnico superior de organización, investigación, enseñanza, asesoramiento y divulgación respecto a las materias encuadradas en su designación".

Desde su creación hasta comienzos de la década de los 50, los pioneros de la Institución comienzan a gestar una idea que a medida que madura va adquiriendo forma de proyecto, editar una publicación periódica que aglutine la producción cientifica del momento sobre la especialidad y sirva como base de la importante misión de divulgación del conocimiento que en dicha materia tienen encomendada, así, en el último trimestre de 1952, se edita el primer número de la revista de "Medicina y Seguridad del Trabajo".

Desde el número 1 hasta el 206 actual, han pasado 55 años, podemos considerar que la publicación se encuentra en su etapa de madurez si tenemos en cuenta que según estudios de la UNESCO, aproximadamente el $80 \%$ de las publicaciones periódicas nuevas no logran alcanzan una vida media de dos años, o en su más tierna infancia, si la comparamos con Philosophical Transactions, revista que nace en 1665, editada por la Royal Society de Londres, y considerada clave en el cambio conceptual más importante y decisivo del mundo cientifico y editorial, al tratarse de la primera publicación en incorporar el proceso de revisión por expertos previo a la publicación.

En su madurez o en su más tierna infancia, lo que es cierto es que aquel proyecto iniciado en los años 50 por un grupo de profesionales del antiguo Instituto Nacional de Medicina y Seguridad del Trabajo, constituye hoy día la consolidación de un éxito editorial, sobre todo, si somos conscientes de que mantener a flote una publicación periódica y, no digamos ya con éxito, no es tarea fácil ni responde a iniciativas individuales. Así, detrás de los pioneros, podemos encontrar una cadena de equipos editoriales que con su esfuerzo han contribuido a mantener la trayectoria de la publicación de forma ininterrumpida hasta nuestros días, por eso, desde este Editorial, y transmitiendo tanto un sentimiento personal como del resto de integrantes del nuevo equipo directivo de la revista, quisiéramos reconocer el esfuerzo con el que cada uno de ellos ha contribuido y agradeciendo la labor que han realizado durante las diferentes edades de la publicación.

En mayor o menor medida, este proceso de adaptación ha jugado un papel importante a la hora de adecuar el grado de integración de la revista en su propio medio y por mantener su interacción con sus lectores, base principal del éxito y lo que ha permitido mantener viva la revista durante los 55 años de su 
existencia. En cada escenario se mueven sus actores, y en esta representación no podemos olvidar el papel decisivo de algunos de ellos, por eso, también es nuestro deseo agradecer a los colaboradores, autores, suscriptores y lectores la confianza depositada en la revista durante todo este tiempo y solicitar la misma para el futuro.

A lo largo de estos años, Medicina y Seguridad del Trabajo ha ido creciendo y adaptándose a las diferentes exigencias profesionales e inquietudes cientificas de los distintos momentos históricos por los que ha ido atravesando. Los permanentes cambios de escenario y sus consecutivas adaptaciones, han ido moldeando el proceso evolutivo a lo largo de toda su trayectoria editorial, y esto nos ayuda a entender la realidad del momento como una parte de un proceso continuo, afrontando nuevos retos como una constante bien definida dentro de la propia línea editorial.

Si echamos una mirada a nuestro entorno, nos encontramos ante un escenario cientifico y editorial que poco tiene que ver con el que se perfilaba en un pasado relativamente reciente. Desde la aparición de Internet a finales del siglo pasado, surge una verdadera revolución de los sistemas de información y gestión del conocimiento, así, la aparición de la edición electrónica y los nuevas herramientas de acceso y difusión de la producción cientifica, suponen un cambio importante de mentalidad de una sociedad cientifica que se mueve dentro de la universalización de las fuentes del conocimiento aprovechando las ventajas que ofrece las Tecnologías de la Comunicación y la Información (TICs).

Nuestro primer deseo al afrontar esta etapa que iniciamos al frente de la publicación, es exponer a todos los profesionales y lectores de la revista, la filosofía con la que pretendemos abordar esta labor al frente de la misma y los retos que nos hemos planteado durante este periodo.

Conscientes de la realidad que vivimos en un mundo editorial inmerso en lo que denominamos sociedad de la información o sociedad digital, los más atrevidos, "era post Gutember", hemos partido de dos premisas como base conceptual del proyecto, la primera, invertir nuestros esfuerzos en adaptar la publicación al competitivo escenario editorial en el que el que nos movemos actualmente y, el segundo, elaborar estrategias que permitan ampliar el rango de difusión y visibilidad de la misma.

Para ello, uno de nuestros objetivos inmediatos, se centra en ampliar la participación de expertos en el Consejo Cientifico, atendiendo a características tanto de prestigio como de pluralidad institucional y geográfica, en lo que se refiere, tanto a la esfera nacional como internacional.

Otro de los aspectos que pretendemos abordar a lo largo de este periodo, es incorporar la revista dentro de los principales circuitos de acceso y difusión internacionales, aprovechando las oportunidades que actualmente se encuentran a nuestro alcance mediante la utilización de herramientas basadas en Tecnologías de la Información y Comunicación (TIC) y usando Internet como telón de fondo.

En este sentido, nuestros primeros esfuerzos se orientarán en el sentido de integrar la revista en la Red Scientific Electronic Library Online (SciELO), herramienta que ha sido desarrollada por la Organización Panamericana de la Salud de la Organización Mundial de la Salud (OPS/OMS) a través de BIREME y que en España opera la Biblioteca Nacional de Ciencias de la Salud del Instituto de Salud Carlos III. En la actualidad, SciELO se encuentra considerado como uno de los principales sistemas internacionales de acceso abierto a texto completo y adicionalmente, una de las plataformas de mayor difusión, con despliegue generalizado entre los países de Latinoamérica.

Por último, y en esta misma dirección, pretendemos abrir líneas de expansión al objeto de que la versión impresa de la revista, pueda llegar a los principales Centros de Conocimiento de las principales 
instituciones relacionadas con el tema en los países de Latinoamérica, sin exclusión de otros países, principalmente europeos y del área mediterránea.

Mediante una estrategia que integra acciones en diferentes direcciones, pretendemos actuar de forma simultanea en distintos ámbitos, de manera que logremos incrementar su rango de difusión, su calidad cientifica y ampliar la oferta de la revista en una doble vertiente, como fuente de acceso a conocimiento y como alternativa de referencia en cuanto a publicación de una comunidad científica más amplia y heterogénea geográficamente.

Retomando la reflexión inicial con la que he comenzado este editorial, cualquier reto supone convivir con sensaciones de expectativa e incertidumbre, entendemos que enfocar el proyecto sin olvidar el pasado, nos ha ayudado a centrar su diseño en el marco actual, pero sobre todo, a pensar con visión de futuro. Ojalá podamos cerrar nuestra etapa tras haber cumplido los objetivos que nos hemos marcado, o al menos un importante número de ellos, de lo que estamos seguros es que todo esfuerzo por conseguirlo, va a merecer la pena.

Jorge Veiga de Cabo Redactor Jefe 